\title{
Preparation of Sustained-Release Suppositories Containing Microencapsulated Indomethacin and Bioavailability of Indomethacin in Rabbits ${ }^{1)}$
}

\author{
TOShIAKI NAKAJIMA, ${ }^{*, a}$ YasuJI TAKAShima, ${ }^{a}$ KIN-ICHI IIDA, ${ }^{a}$ \\ HaRUMi Mitsuta, ${ }^{a}$ Atsushi Furuya ${ }^{a}$ and MASUMI KoISHI ${ }^{b}$ \\ Research Center, Taisho Pharmaceutical Co., Ltd., ${ }^{a}$ 1-403, Yoshino-cho, Omiya, Saitama 330, \\ Japan and Faculty of Pharmaceutical Sciences and Institute of Colloid and \\ Interface Science, Science University of Tokyo, ${ }^{b} 12$ Ichigaya \\ Funagawara-machi, Shinjuku-ku, Tokyo 162, Japan
}

(Received February 17, 1987)

\begin{abstract}
The absorption of indomethacin (IM) from suppositories containing surface-modified IM microcapsules (MC) after rectal administration in rabbits was investigated with the aim of producing sustained-release suppositories. The IM-MC were prepared by phase separation of ethylcellulose (EC) from cyclohexane, and the IM surface was modified with a carboxy-vinyl polymer (Hiviswako ${ }^{\circledR} 104$, HW) by a dry blend technique before encapsulation.

As a sustained plasma level of IM was not obtained when IM-MC-containing suppositories which showed a zero-order release profile were administered, the factors affecting the IM absorption, that is, the EC and HW contents in the MC, and the suppository base and type, were investigated. The EC and HW contents in the MC affected the IM release rate but had little effect on the IM absorption. When the IM-MC were directly administered, the IM plasma level was significantly lower than that after administration of the macrogol base suppository containing the IM-MC. The area under the concentration-time curve $(A U C)$ in the case of the Witepsol ${ }^{\circledR}$ base was smaller than that in the case of the macrogol one. The plasma concentration-time curve of the hollow-type suppository showed a lag time, and the $T_{\max }$ was delayed by $1 \mathrm{~h}$ compared with that of the conventional suppository. Thus, it was found that a suitable kind and amount of suppository base and an appropriate suppository type should be selected to prepare sustained-release suppositories containing IM-MC.
\end{abstract}

Keywords__ indomethacin; microcapsule; suppository; bioavailability; rabbit; sustained release; suppository base; surface modification

In the previous paper, ${ }^{2)}$ we reported the preparation of sustained-release suppositories containing surface-modified indomethacin (IM) microcapsules (MC) and the release profile of IM from the suppository was shown to be zero-order. The IM surface was modified with a carboxy-vinyl polymer (Hiviswako ${ }^{\circledR} 104, \mathrm{HW}$ ) by a dry blend technique before encapsulation with ethylcellulose (EC) as a wall material. In this study, we investigated the absorption of IM from suppositories containing IM-MC in rabbits. Since there are some reports ${ }^{3-7)}$ on the effects of suppository bases on drug absorption in animals, we used not only the macrogol base but also the Witepsol ${ }^{\circledR}$ base. In addition, hollow-type ${ }^{3)}$ as well as conventional suppositories were used to investigate the effect of suppository type on the IM absorption in rabbits.

\section{Experimental}

Materials - The sources of materials used in this work were as follows: IM from Sumitomo Chemical Co., Ltd., EC (standard type, $100 \mathrm{cP}$ ) from Hercules Co., Ltd., polyethylene (Sun Wax ${ }^{\circledR}$ 131P, M.W. 3500) from Sanyo Kasei Co., Ltd., and HW from Wako Pure Chemical Industries Ltd. All other chemicals were reagent-grade commercial products. 
Surface Modification of IM- This procedure was carried out as reported previously. ${ }^{2)}$ Thirty grams of IM and either 15 or $30 \mathrm{~g}$ of HW were mixed using an automatic ceramic mortar (Yamato-Nitto, Labo-mill UT-21, Yamato Kagaku Co., Ltd.) for $1 \mathrm{~h}$ at room temperature.

Preparation of the IM-MC — The IM-MC were prepared as reported previously. ${ }^{2)}$ Thirty grams of HWmodified IM $[\mathrm{HW} / \mathrm{IM}=1 / 2$ or $1 / 1(\mathrm{w} / \mathrm{w})], 6-8 \mathrm{~g}$ of EC and cyclohexane $(300 \mathrm{ml})$ containing $1 \%(\mathrm{w} / \mathrm{v})$ polyethylene were placed in a $500 \mathrm{ml}$ flask. The flask was heated to $80-82^{\circ} \mathrm{C}$, then cooled to $40^{\circ} \mathrm{C}$ with continuous stirring $\left(324 \mathrm{rpm}\right.$ ) for $60 \mathrm{~min}$, and finally cooled quickly to $25^{\circ} \mathrm{C}$. The microcapsules that formed were recovered by decantation, washed with cyclohexane and dried under reduced pressure. The MC were passed through JIS standard sieves. ${ }^{2)}$ The $\mathrm{MC}$ with a particle size range of 177 to $250 \mu \mathrm{m}$ were used in the subsequent experiments.

Determination of IM Content - The IM content in IM-MC was determined spectrophotometrically. ${ }^{2)}$ The EC content was obtained by applying the following equation: $\mathrm{EC}(\%)=[(\mathrm{MC}-\mathrm{IM}-\mathrm{HW}) / \mathrm{MC}] \times 100$ where $\mathrm{MC}, \mathrm{IM}$ and HW are the weights of the substances. When IM and HW in the IM-MC were extracted with $0.2 \mathrm{M}$ phosphate buffer and then the wall of the IM-MC was dried, the weight of the wall was almost the same as that obtained from the above equation. Thus, the weight of HW was calculated from its ratio to IM. The values of EC content of the MC were $20.1 \pm 0.9$ (S.D.) $\%$, and $13.9 \pm 1.0$ (S.D.) $\%$ for the HW-modified IM-MC [EC $20 \%, \mathrm{HW} / \mathrm{IM}=1 / 1$ (w/w)], [IM$\mathrm{MC}(\mathrm{I})]$ and $[\mathrm{EC} 14 \%, \mathrm{HW} / \mathrm{IM}=1 / 2(\mathrm{w} / \mathrm{w})]$, [IM-MC (II)], respectively.

Preparation of Conventional and Hollow-Type Suppositories_-Table I shows the suppository formulae. The conventional suppositories were made by the fusion method. ${ }^{2)}$ The bases (about $45 \mathrm{~g}$ ) were fused in a beaker on an oil bath at $70^{\circ} \mathrm{C}$ and cooled to $50^{\circ} \mathrm{C}$. Then, intact IM or IM-MC (1.5 g IM) was added to the bases and dispersed by stirring for $30 \mathrm{~min}$. The fused bases containing intact IM or IM-MC were poured into suppository molds $(1.5 \mathrm{ml}$ in volume), which were quickly placed in a refrigerator at $5{ }^{\circ} \mathrm{C}$. The hollow-type suppositories (macrogol base) were made by the method of Matsumoto et al. ${ }^{3)}$ The bases without IM-MC were melted and poured into smaller suppository containers $(1.0 \mathrm{ml}$ in volume). The containers were cooled to form hollow suppositories, IM-MC (IM $25 \mathrm{mg}$ ) were put into the hollow part, and the same melted bases were added to complete the hollow-type suppositories.

Release of IM from Suppositories-Release of IM was measured by the method of Muranishi et al. ${ }^{8)}$ as described previously. ${ }^{2)}$ The test solution was $0.2 \mathrm{M}$ phosphate buffer solution ( $\mathrm{pH} 7.2$ ). A suppository was placed in $3 \mathrm{ml}$ of the test solution in a cylindrical cell equipped with a Millipore filter (pore size $3.0 \mu \mathrm{m}$ ) and stirred with a rod at $25 \mathrm{rpm}$. The cell was connected with a glass vessel containing $300 \mathrm{ml}$ of the test solution which was stirred with a magnetic stirrer at $100 \mathrm{rpm}$. The IM concentration was assayed spectrophotometrically at $318 \mathrm{~nm}$.

Animal Experiments-White male rabbits each weighing $2.8-3.6 \mathrm{~kg}$ were fasted for $48 \mathrm{~h}$ prior to the experiments but allowed free access to water. About a half of the conventional suppository or about three-quarters of the hollow-type suppository ( $750 \mathrm{mg}, 25 \mathrm{mg}$ content of IM) was inserted by hand. The IM-MC (IM $25 \mathrm{mg}$ ) was directly inserted with a $2 \mathrm{ml}$ syringe whose needle had been cut off. Retention of the suppositories and IM-MC by the rabbits was ensured by fastening the anus with a clip after insertion. Blood $(2 \mathrm{ml})$ was taken by cardiac puncture from rabbits at different time intervals. The plasma was obtained by centrifugation at $3000 \mathrm{rpm}$ for $10 \mathrm{~min}$.

Assay of IM in Plasma_- The plasma $(0.7 \mathrm{ml})$ was pipeted into a glass stoppered centrifuge tube containing $2 \mathrm{ml}$ of $0.2 \mathrm{M}$ citrate buffer ( $\mathrm{pH} \mathrm{3.6)}$ and $10 \mathrm{ml}$ of ethyl acetate. The test tube was mechanically shaken for $10 \mathrm{~min}$ and then centrifuged at $3000 \mathrm{rpm}$ for $10 \mathrm{~min}$. Eight milliliters of the ethyl acetate phase was pipeted into the other centrifuge tube and evaporated to dryness under reduced pressure. The residue was dissolved in $250 \mu \mathrm{l}$ of a mobile phase. A $20 \mu \mathrm{l}$ sample was injected into the high performance liquid chromatography apparatus (Hitachi 655-12 liquid chromatograph with a Hitachi $655 \mathrm{~A}$ variable-wavelength UV monitor). The conditions for analysis were as

TABLE I. Formulae of Suppositories

\begin{tabular}{|c|c|c|c|}
\hline \multicolumn{2}{|l|}{ Macrogol type } & \multicolumn{2}{|l|}{ Witepsol ${ }^{\circledR}$ type } \\
\hline Component & $\mathrm{mg}$ & Component & $\mathrm{mg}$ \\
\hline Microencapsulated IM & $50.0^{a)}$ & Microencapsulated IM & $50.0^{a)}$ \\
\hline Glycerine & 120.0 & Dibutylhydroxytoluene & 1.2 \\
\hline Dibutylhydroxytoluene & 0.8 & Light anhydrous silicic acid & 30.0 \\
\hline Distilled water & 30.0 & Witepsol ${ }^{\circledR}$ E85 & 210.0 \\
\hline Hydrogenated castor oil & 6.0 & Witepsol ${ }^{\circledR}$ S55 & 1500.0 \\
\hline $\mathrm{POE}^{b)}(40)$ monostearate & 30.0 & & \\
\hline Macrogol 1540 & 13.0 & & \\
\hline Macrogol 6000 & 64.0 & & \\
\hline Macrogol 4000 & 1500.0 & & \\
\hline
\end{tabular}

a) IM content. b) Polyoxyethylene. 
follows: column, $15 \mathrm{~cm} \times 4 \mathrm{~mm}$ i.d.; packing, TSK-LS $410(5 \mu \mathrm{m})$ ODS; mobile phase, methanol-water-acetic acid-triethanolamine $(74.3: 25: 0.5: 0.2)$; flow rate, $0.5 \mathrm{ml} / \mathrm{min}$; wavelength, $\mathrm{UV}$ at $260 \mathrm{~nm}$; column temperature, $50^{\circ} \mathrm{C}$.

\section{Results and Discussion}

\section{Release and Absorption Studies on Suppositories Containing IM-MC (I)}

In the release test, the conventional macrogol base suppository containing IM-MC (I) gave a zero-order release profile, as shown in Fig. 1A. In the absorption test, however, the IM plasma level was not well sustained, as shown in Fig. 2A and Table II. The area under the concentration-time curve $(A U C)$ was calculated by means of the trapezoidal method. The $A U C_{0}^{8}$ value after administration of the suppositories containing IM-MC (I) was $26.91 \mu \mathrm{g} \cdot \mathrm{h} / \mathrm{ml}$ and it was about $76 \%(p<0.05)$ of the value of the suppositories containing intact IM. Although the suppositories containing IM-MC (I) showed a zero-order release profile in the release test, the IM plasma concentration was not well sustained; $T_{\max }$ was delayed only 30 min compared with the suppositories containing intact IM. These results

A
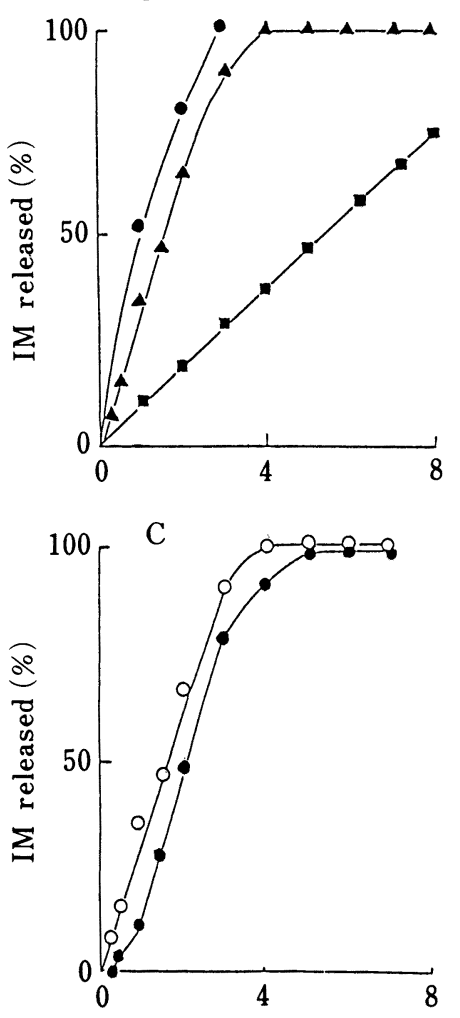

Time (h)
B
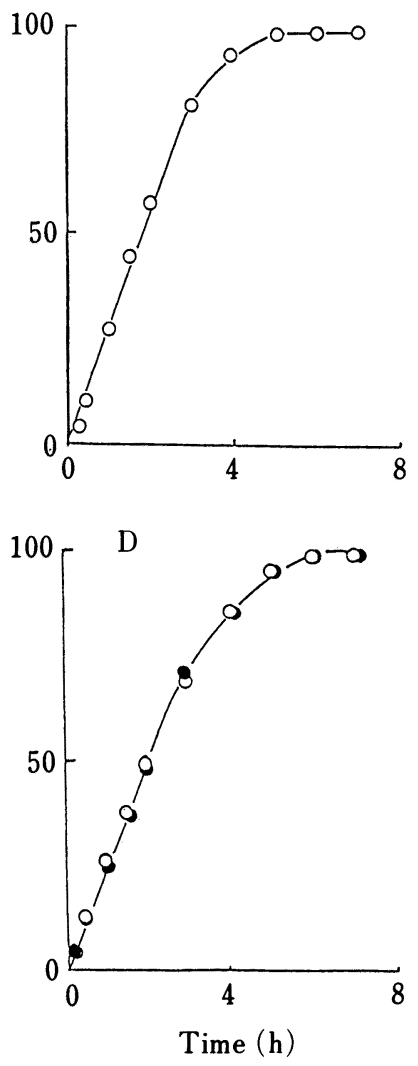

Fig. 1. Release Profiles of IM from Various Suppositories and IM-MC (II)

A: Conventional-type macrogol base suppositories. Content: $\bigcirc$, intact IM; $\square$, IM-MC

(I) $(\mathrm{EC} 20 \%, \mathrm{HW} / \mathrm{IM}=1 / 1) ; \Delta$, IM-MC (II) $(\mathrm{EC} 14 \%, \mathrm{HW} / \mathrm{IM}=1 / 2)$.

B: IM-MC (II), O.

C: Hollow- and conventional-type macrogol base suppositories containing IM-MC (II).

Type: 0 , hollow; $\bigcirc$, conventional. (II).

D: Conventional-type Witepsol ${ }^{\circledR}$ base suppositories. Content: 9 , intact IM; O, IM-MC

Each value represents the mean $(n=2)$. 

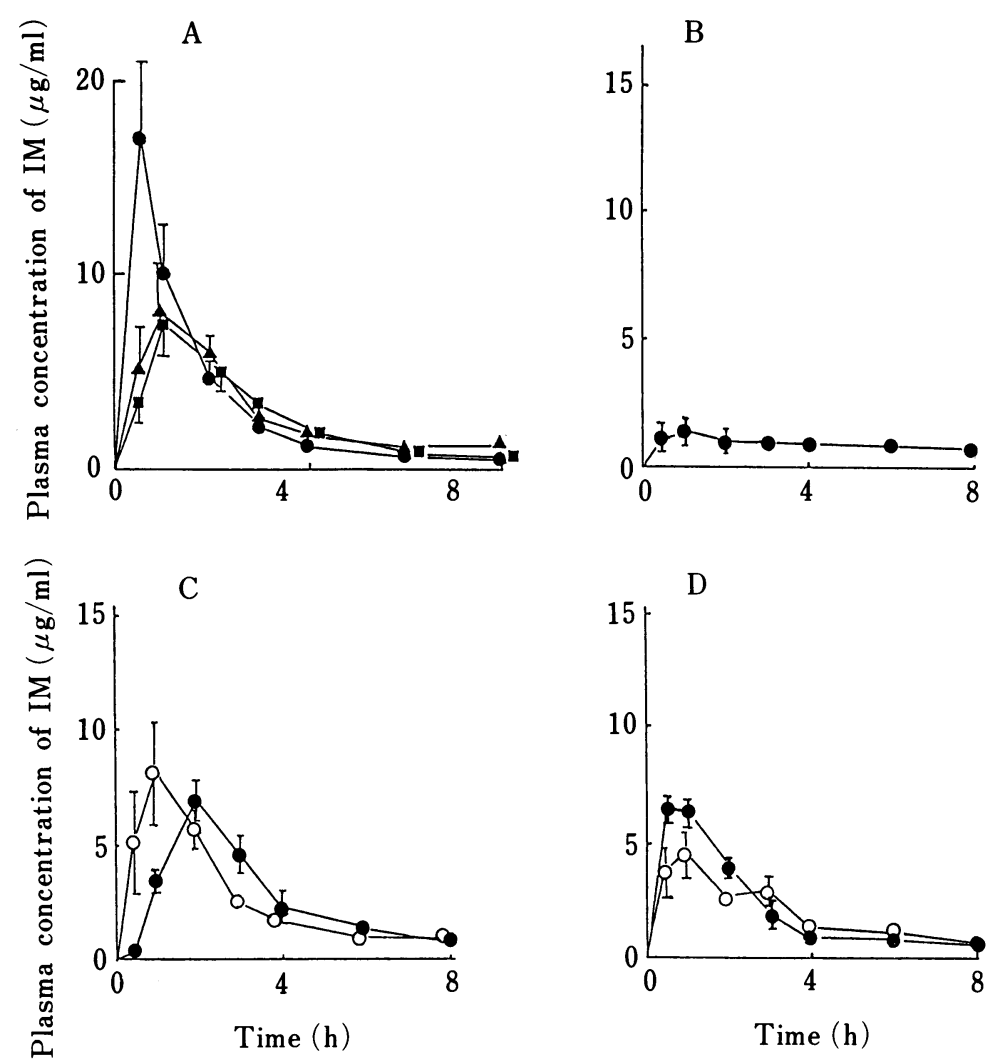

Fig. 2. Plasma Concentration of IM after Rectal Administration of Various Suppositories and IM-MC (II)

A: Conventional-type macrogol base suppositories. Content: $\bullet$, intact IM; $\mathbf{\square}$, IM-MC

(I) $(\mathrm{EC} 20 \%, \mathrm{HW} / \mathrm{IM}=1 / 1) ; \boldsymbol{\Lambda}$, IM-MC (II) $(\mathrm{EC} 14 \%, \mathrm{HW} / \mathrm{IM}=1 / 2)$.

B: IM-MC (II),

C: Hollow- and conventional-type macrogol base suppositories containing IM-MC (II).

Type: $\bullet$, hollow; $\bigcirc$, conventional.

D: Conventional-type Witepsol ${ }^{\circledR}$ base suppositories. Content: $\bullet$, intact IM; O, IM-MC (II).

Each value represents the mean \pm S.E. $(n=3-6)$.

suggest that the amount of the rabbit rectal fluid was less than that of the medium of the release test, and thus not all of the IM in IM-MC was released in the rabbit rectum.

Release and Absorption Studies on Conventional Macrogol Base Suppositories Containing IMMC (II)

To obtain sufficient release of IM from the suppositories in the rabbit rectum, the EC and HW contents were reduced when the MC was prepared. Figures $1 \mathrm{~A}$ and $2 \mathrm{~A}$ and Table II also show the results of the release and absorption tests of the macrogol base suppositories containing IM-MC (II). The rate of the release of IM from the suppositories containing IMMC (II) was faster than that from the suppositories containing IM-MC (I), and the time required to release $50 \%$ of IM from the suppository $\left(t_{50}\right)$ was about $90 \mathrm{~min}$. Umeda et al..$^{9)}$ reported a sustained-release suppository containing microencapsulated IM with gelatin as a wall material. They found that the IM plasma level was sustained when a suppository with a $t_{50}$ of about $90 \mathrm{~min}$ was administered to a rabbit, although the release test was not exactly the same as in our experiment. Thus, we used IM-MC (II) to obtain complete release of IM from the suppositories in the rabbit rectum. As shown in Fig. 2B, however, the IM plasma levels of 
TABLE II. Pharmacokinetic Parameters of IM after Rectal Administration in Rabbits

\begin{tabular}{|c|c|c|c|c|c|c|c|}
\hline Base & Type & Containing & $\underset{(\mu \mathrm{g} / \mathrm{ml})}{C_{\max }}$ & $\begin{array}{c}T_{\max } \\
\text { (h) }\end{array}$ & $\begin{array}{c}A U C_{0}^{8} \\
(\mu \mathrm{g} \cdot \mathrm{h} / \mathrm{ml})\end{array}$ & $\begin{array}{l}\mathrm{BA}^{d)} \\
(\%)\end{array}$ & $n$ \\
\hline \multirow[t]{4}{*}{ Macrogol } & \multirow[t]{3}{*}{ Conventional } & Intact IM & $17.11 \pm 3.91$ & 0.5 & $26.91 \pm 5.42$ & 100 & 6 \\
\hline & & $\mathrm{IM}-\mathrm{MC}(\mathrm{I})^{a)}$ & $7.27 \pm 1.61$ & 1 & $20.70 \pm 0.81$ & 76.9 & 3 \\
\hline & & $\mathrm{IM}-\mathrm{MC}(\mathrm{II})^{b)}$ & $9.07 \pm 1.63$ & 1 & $23.45 \pm 2.52$ & 87.1 & 3 \\
\hline & Hollow & IM-MC (II) & $6.83 \pm 0.93$ & 2 & $21.13 \pm 3.17$ & 78.5 & 3 \\
\hline \multirow[t]{2}{*}{ Witepsol ${ }^{\circledR}$} & \multirow[t]{2}{*}{ Conventional } & Intact IM & $6.61 \pm 0.62$ & 0.5 & $17.50 \pm 0.84$ & 65.0 & 3 \\
\hline & & IM-MC (II) & $4.97 \pm 0.73$ & 1 & $15.87 \pm 0.38$ & 59.0 & 3 \\
\hline Microcapsule ${ }^{c)}$ & & IM-MC (II) & $1.26 \pm 0.64$ & 1 & $5.66 \pm 2.20$ & 21.0 & 3 \\
\hline
\end{tabular}

a) HW-modified IM-MC (EC $20 \%, \mathrm{HW} / \mathrm{IM}=1 / 1) . \quad b)$ HW-modified IM-MC (EC 14\%, HW/IM =1/2). c) IM-MC (II) were directly administered into the rectum. d) BA, the ratio to the $A U C_{0}^{8}$ of the conventional-type macrogol base suppository containing intact IM. Each value of $C_{\max }$ and $A U C_{0}^{8}$ represents the mean \pm S.E. $(n=3-6)$.

the suppositories containing IM-MC (II) were not well sustained, and the $A U C_{0}^{8}$ did not increase significantly compared with the case of IM-MC (I). The reduction in EC and HW contents in IM-MC (II) did not result in a sustained IM plasma level after rectal administration.

\section{Release and Absorption Studies on IM-MC (II)}

The IM release test and the IM absorption test were carried out with IM-MC (II) to avoid the effect of suppository bases. Figures $1 \mathrm{~B}$ and $2 \mathrm{~B}$ and Table II show the results. The release rates were almost the same as those of the suppositories containing IM-MC (II). On the other hand, the IM plasma levels showed a sustained-release pattern, although the IM plasma levels and the $A U C_{0}^{8}$ were lower and smaller than those of the suppositories containing IM-MC (II). These results indicate that the IM release was suppressed by the microencapsulation of IM, but that the suppository bases considerably affected the IM release and absorption in the rabbit rectum.

\section{Release and Absorption Studies on Hollow-Type Suppositories Containing IM-MC (II)}

The IM plasma levels of the suppositories containing IM-MC (II) were higher than those of IM-MC (II), as shown in Fig. 2A and 2B. One of the reasons was thought to be the effect of the suppository base on the IM absorption. Another reason might be that part of the IM in the IM-MC (II) was released in the suppository base while the suppositories were being prepared. Thus, the application of the hollow-type suppository was investigated to eliminate the putative release of IM into the suppository base during preparation. Figures $1 \mathrm{C}$ and $2 \mathrm{C}$ and Table II show the results of the release and absorption studies of hollow-type macrogol base suppositories containing IM-MC (II). The IM release profiles of the hollow-type suppositories showed a short lag time but were not very different from those of the conventional suppositories containing IM-MC (II); the $t_{50}$ was delayed about $30 \mathrm{~min}$ compared with that of the conventional suppositories.

The IM plasma concentration-time curves of the hollow-type suppositories also showed a lag time corresponding to the release profiles. The $T_{\max }$ of the hollow-type and the conventional suppositories were 2 and $1 \mathrm{~h}$, respectively. The amount of release of IM from IM-MC (II) while the suppositories were being prepared was not considered to be significant because the $A U C_{0}^{8}$ of the conventional suppositories containing IM-MC (II) was not significantly different from that of the hollow-type ones, and the patterns of the IM plasma concentration-time curves were almost the same except for the lag time. The hollow-type suppositories containing IM-MC (II) gave a delayed $T_{\max }$ in the absorption test. 


\section{Release and Absorption Studies on Conventional-Type Witepsol ${ }^{\circledR}$ Base Suppositories Containing IM-MC (II)}

Watanabe et $a l .^{3)}$ reported that the IM serum level obtained with the water-soluble base suppository was higher than that with the Witepsol ${ }^{\circledR}$ base suppository, and Vidras et al. ${ }^{6)}$ reported that the IM serum level obtained with a Witepsol ${ }^{\circledR}$ base suppository was higher than that with a macrogol base suppository up to $30 \mathrm{~min}$ after rectal administration. Thus, Witepsol ${ }^{\circledR}$ base suppositories containing IM-MC (II) were also examined. Figures $1 \mathrm{D}$ and $2 \mathrm{D}$ and Table II show the results of the release and absorption tests on Witepsol ${ }^{\circledR}$ base suppositories containing intact IM and IM-MC (II). The IM release profile of the Witepsol ${ }^{\circledR}$ base suppository containing IM-MC (II) was almost the same as that of the suppository containing intact IM. The IM release rate from the Witepsol ${ }^{\circledR}$ base suppositories containing intact IM or IM-MC (II) was somewhat slower than that from the macrogol base suppositories, as shown in Fig. 1A. The IM plasma level of the Witepsol ${ }^{\circledR}$ base suppositories containing IM-MC (II) was lower than that of the suppositories containing intact IM and was not well sustained; $T_{\max }$ was delayed only $30 \mathrm{~min}$. The $A U C_{0}^{8}$ of the Witepsol ${ }^{\circledR}$ base suppositories was lower $(p<0.05)$ than that of the macrogol base ones containing intact IM or IM-MC (II). These results correspond to those of Watanabe et al., ${ }^{3)}$ but not to those of Vidras et al. ${ }^{6)}$

Tsuchiya et al. ${ }^{7)}$ reported that the secretion of the rectal fluid varied with the kind and amount of the suppository base; the rectal fluid volume increased with increase in the amount of the macrogol base, and there was no detectable amount of fluid but some mucus in the rectum after administration of an oleaginous base suppository.

These findings help to explain our experimental results. As hardly any rectal fluid was secreted, the IM plasma level and the $A U C_{0}^{8}$ were low when IM-MC (II) and the Witepsol ${ }^{\circledR}$ base suppository containing IM-MC (II) were administered. On the other hand, when the macrogol base suppository containing IM-MC (II) was administered, enough rectal fluid was secreted. Thus the IM plasma level was high because IM was released well. It seems clear that when IM-MC are used to prepare sustained-release suppositories, a suitable kind and amount of suppository base and an appropriate suppository type should be selected. For instance, the combinations of conventionl- and hollow-type suppositories with Witepsol ${ }^{\circledR}$ and macrogol base could be useful for the production of sustained-release suppositories containing HWmodified IM-MC.

\section{References and Notes}

1) This work was presented at the 2nd Annual Meeting of the Academy of Pharmaceutical Science and Technology of Japan, Tokyo, September 1986.

2) T. Nakajima, Y. Takashima, K. Iida, H. Mitsuta and M. Koishi, Chem. Pharm. Bull, 35, 1201 (1987).

3) Y. Watanabe, K. Yokoyama, M. Yamaji, F. Tanaka and M. Matsumoto, Yakugaku Zasshi, 105, 278 (1985); Y. Watanabe, Y. Tone, M. Suzuki and M. Matsumoto, ibid., 105, 284 (1985).

4) T. Ogiso, M. Iwaki and E. Tamaki, J. Pharmacobio-Dyn., 7, 392 (1984).

5) T. Fukuda, Y. Takeuchi, Y. Hanyu, M. Yamashita, I. Kaneko, K. Tahira and Y. Fukumori, Yakuzaigaku, 40, 167 (1980).

6) N. J. Vidras, V. E. Reid, N. R. Bohidar and F. M. Plakogiannis, J. Pharm. Sci., 71, 945 (1982).

7) S. Tsuchiya, M. Hiura and H. Matsumaru, Chem. Pharm. Bull., 25, 667 (1977).

8) S. Muranishi, Y. Okubo and H. Sezaki, Yakuzaigaku, 39, 1 (1979).

9) T. Umeda, A. Matsuzawa, T. Yokoyama, K. Kuroda and T. Kuroda, Chem. Pharm. Bull., 31, 2793 (1983); T. Kuroda, T. Yokoyama, T. Umeda, A. Matsuzawa, K. Kuroda and S. Asada, ibid., 31, 3319 (1983). 